\title{
On Indistinguishability Operators, Fuzzy Metrics and Modular Metrics
}

\author{
Juan-José Miñana (iD and Oscar Valero * \\ Department of Mathematics and Computer Science, Universitat de les Illes Balears, \\ Carretera de Valldemossa km. 7.5, 07122 Palma, Spain; jj.minana@uib.es \\ * Correspondence: o.valero@uib.es; Tel.: +34-971-259817
}

Received: 20 November 2017; Accepted:12 December 2017; Published: 15 December 2017

\begin{abstract}
The notion of indistinguishability operator was introduced by Trillas, E. in 1982, with the aim of fuzzifying the crisp notion of equivalence relation. Such operators allow for measuring the similarity between objects when there is a limitation on the accuracy of the performed measurement or a certain degree of similarity can be only determined between the objects being compared. Since Trillas introduced such kind of operators, many authors have studied their properties and applications. In particular, an intensive research line is focused on the metric behavior of indistinguishability operators. Specifically, the existence of a duality between metrics and indistinguishability operators has been explored. In this direction, a technique to generate metrics from indistinguishability operators, and vice versa, has been developed by several authors in the literature. Nowadays, such a measurement of similarity is provided by the so-called fuzzy metrics when the degree of similarity between objects is measured relative to a parameter. The main purpose of this paper is to extend the notion of indistinguishability operator in such a way that the measurements of similarity are relative to a parameter and, thus, classical indistinguishability operators and fuzzy metrics can be retrieved as a particular case. Moreover, we discuss the relationship between the new operators and metrics. Concretely, we prove the existence of a duality between them and the so-called modular metrics, which provide a dissimilarity measurement between objects relative to a parameter. The new duality relationship allows us, on the one hand, to introduce a technique for generating the new indistinguishability operators from modular metrics and vice versa and, on the other hand, to derive, as a consequence, a technique for generating fuzzy metrics from modular metrics and vice versa. Furthermore, we yield examples that illustrate the new results.
\end{abstract}

Keywords: indistinguishability operator; fuzzy (pseudo-)metric; modular (pseudo-)metric; continuous Archimedean $t$-norm; additive generator; pseudo-inverse

\section{Introduction}

Throughout this paper, we will use the following notation. We will denote by $\mathbb{R}$ the set of real numbers, and we will denote by $[a, b],] a, b],[a, b[$ and $] a, b[$, open, semi-open and closet real intervals, respectively, whenever $a, b \in \mathbb{R} \cup\{-\infty, \infty\}$ with $a<b$.

In 1982, Trillas, E. introduced the notion of indistinguishability operator with the purpose of fuzzifying the classical (crisp) notion of equivalence relation (see [1]). Let us recall that an indistinguishability operator, for a $t$-norm $*$, on a non-empty set $X$ is a fuzzy set $E: X \times X \rightarrow[0,1]$, which satisfies, for each $x, y, z \in X$, the following axioms:

(E1) $E(x, x)=1$;

(Reflexivity)

(E2) $E(x, y)=E(y, x)$;

(Symmetry)

(E3) $E(x, y) * E(y, z) \leq E(x, z)$.

(Transitivity)

If, in addition, $E$ satisfies for all $x, y \in X$ the following condition: 
(E1') $E(x, y)=1$ implies $x=y$,

then it is said that $E$ separates points.

According to [1] (see also [2]), the numerical value $E(x, y)$ provides the degree up to which $x$ is indistinguishable from $y$ or equivalent to $y$. Thus, the greater $E(x, y)$, the more similar $x$ and $y$ are. In particular, $E(x, y)=1$ when $x=y$.

In the light of the preceding definition, the concept of $t$-norm plays an essential role in the framework of indistinguishability operators. In fact, $t$-norms are involved in axiom (E3) in order to express that $x$ is indistinguishable from $z$ whenever $x$ is indistinguishable from $y$ and $z$. Throughout this paper, we will assume that the reader is familiar with the basics of triangular norms (see [3] for a deeper treatment of the topic).

Since Trillas introduced the indistinguishability operators, many authors have studied their properties and applications. We refer the reader to [2], and references therein, for an exhaustive treatment of the topic. Among the different properties that such operators enjoy, the metric behavior can be highlighted. In particular, the existence of a duality relationship between metrics and indistinguishability operators in [2-8] has been explored. In this direction, a technique to generate metrics from indistinguishability operators, and vice versa, has been developed by several authors in the literature. Concretely, an indistinguishability operator can be provided from a (pseudo-)metric as follows:

Theorem 1. Let $X$ be a non-empty set and let $*$ be a $t$-norm with additive generator $f_{*}:[0,1] \rightarrow[0, \infty]$. If $\diamond$ is a t-norm, then the following assertions are equivalent:

(1) $* \leq \diamond$ (i.e., $x * y \leq x \diamond y$ for all $x, y \in[0,1]$ ).

(2) For any indistinguishability operator $E$ on $X$ for $\diamond$, the function $d^{E, f_{*}}: X \times X \rightarrow[0, \infty]$ defined, for each $x, y \in X, b y$

$$
d^{E, f_{*}}(x, y)=f_{*}(E(x, y))
$$

is a pseudo-metric on X.

(3) For any indistinguishability operator $E$ on $X$ for $\diamond$ that separates points, the function $d^{E, f_{*}}: X \times X \rightarrow[0, \infty]$ defined, for each $x, y \in X$, by

$$
d^{E, f_{*}}(x, y)=f_{*}(E(x, y))
$$

is a metric on $X$.

Reciprocally, a technique to construct an indistinguishability operator from a (pseudo-)metric can be given as the next result shows.

Theorem 2. Let $X$ be a non-empty set and let $*$ be a continuous $t$-norm with additive generator $f_{*}:[0,1] \rightarrow[0, \infty]$. If $d$ is a pseudo-metric on $X$, then the function $E^{d, f_{*}}: X \times X \rightarrow[0,1]$ defined, for all $x, y \in X$, by

$$
E^{d, f_{*}}(x, y)=f_{*}^{(-1)}(d(x, y))
$$

is an indistinguishability operator for $*$, where $f_{*}^{(-1)}$ denotes the pseudo-inverse of the additive generator $f_{*}$. Moreover, the indistinguishability operator $E^{d, f_{*}}$ separates points if and only if $d$ is a metric on X.

It must be stressed that, in the statement of the preceding results, and, throughout this paper, the considered (pseudo-)metrics can take the value $\infty$, which are also known as extended (pseudo-)metrics in [9].

Recently, applications of the techniques exposed in Theorems 1 and 2 to the task allocation problem in multi-agent (multi-robot) systems have been given in [10-12]. In particular, in the preceding references, indistinguishability operators have shown to be appropriate to model response functions when response threshold algorithms (in swarm-like methods) are under consideration in order to solve the aforesaid task allocation problem. 
Nowadays, in many applications, the degree of similarity is measured relative to a parameter (see, for instance, [13-15]). In this case, the indistinguishability operators are not able to measure such a graded similarity and so a new measurement becomes indispensable instead. The aforesaid measurements are called fuzzy metrics and they were introduced in 1975 by Kramosil, I. and Michalek, J. in [16]. However, currently, the fuzzy metric axioms used in the literature are those given by Grabiec, M. in [17] and by George, A. and Veeramani, P. in [18]. It must be pointed out that the axioms by Grabiec and by George and Veeramani, P. are just a reformulation of those given by Kramosil and Michalek.

Let us recall, on account of $[17,18]$, that a fuzzy metric on a non-empty set $X$ is a pair $(M, *)$ such that $*$ is a continuous $t$-norm and $M$ is a fuzzy set on $X \times X \times[0, \infty[$, satisfying the following conditions, for all $x, y, z \in X$ and $s, t>0$ :

(KM1) $M(x, y, 0)=0$;

(KM2) $M(x, y, t)=1$ for all $t>0$ if and only if $x=y$;

(KM3) $M(x, y, t)=M(y, x, t)$;

(KM4) $M(x, y, t) * M(y, z, s) \leq M(x, z, t+s)$;

(KM5) The function $M_{x, y}:\left[0, \infty\left[\rightarrow[0,1]\right.\right.$ is left-continuous, where $M_{x, y}(t)=M(x, y, t)$.

Similar to the classical case, we will say that $(M, *)$ is a fuzzy pseudo-metric on $X$ provided that axiom (KM2) is replaced by the following weaker one:

(KM2') $M(x, x, t)=1$ for all $t>0$.

Moreover, given a fuzzy (pseudo-)metric $(M, *)$ on $X$, we will also say that $(X, M, *)$ is a fuzzy (pseudo-)metric space.

According to [18], the numerical value $M(x, y, t)$ yields the degree of similarity between $x$ and $y$ relative to the value $t$ of the parameter. Of course, it must be clarify that, according to the exposed interpretation, axiom (KM1) does not provide any information from a measurement framework because the rest of axioms are enough in order to define a fuzzy measurement. Motivated by this fact, we will assume that a fuzzy metric $(M, *)$ is a fuzzy set $M$ on $X \times X \times] 0, \infty[$ that satisfies all the preceding axioms except the axiom (KM1). Of course, the left-continuity of axiom (KM5) will be satisfied for the the function $\left.M_{x, y}:\right] 0, \infty[\rightarrow[0,1]$.

The following is a well-known example of fuzzy metric.

Example 1. Let $d$ be a metric on a non-empty set $X$. Let $M_{d}$ be a fuzzy set on $\left.X \times X \times\right] 0, \infty[$ defined, for each $x, y \in X, b y$

$$
M_{d}(x, y, t)=\frac{t}{t+d(x, y)},
$$

whenever $t>0$. On account of [18], $\left(M_{d}, \wedge\right)$ is a fuzzy metric on $X$, where $\wedge$ denotes the minimum $t$-norm. The fuzzy metric $M_{d}$ is called the standard fuzzy metric induced by $d$.

Following [19], a fuzzy metric $(M, *)$ is said to be stationary provided that the function $\left.M_{x, y}:\right] 0, \infty\left[\rightarrow[0,1]\right.$ defined by $M_{x, y}(t)=M(x, y, t)$ is constant for each $x, y \in X$.

The next example gives an instance of stationary fuzzy metric.

Example 2. Let $X$ be a non-empty set $X$ and let $G: X \times X \rightarrow] 0, \frac{1}{2}[$ be a function such that $G(x, y)=G(y, x)$ for all $x, y \in X$. Consider the fuzzy set $M_{G}$ on $\left.X \times X \times\right] 0, \infty\left[\right.$ given by $M_{G}(x, y, t)=G(x, y)$ for all $t>0$ and $x, y \in X$ such that $x \neq y$ and $M_{G}(x, x, t)=1$ for all $t>0$. According to [13], $\left(M_{G}, *_{L}\right)$ is a stationary fuzzy metric, where $*_{L}$ is the Luckasievicz $t$-norm.

Notice that, as in the case of indistinguishability operators, $t$-norms are crucial in the definition of a fuzzy metric. However, now the unique $t$-norms under consideration are the continuous ones. Thus, it constitutes a considerable difference between indistinguishability operators and fuzzy metrics. Moreover, another significant difference between these two kinds of fuzzy measurement is that 
fuzzy metrics include in their definition a parameter. Therefore, none of these types of similarity measurements generalizes the other.

In the light of the preceding fact, it seems natural to try to unify both notions, fuzzy (pseudo-)metrics and indistinguishability operators, under a new one. Thus, the aim of this paper is twofold. On the one hand, we introduce a new type of operator, which we have called modular indistinguishability operator (the name will be justified in Section 3), which provides a degree of similarity or equivalence relative to a parameter and retrieves as a particular case fuzzy (pseudo-)metrics and classical indistinguishability operators. On the other hand, we explore the metric behavior of this new kind of operators. Specifically, we study the duality relationship between modular indistinguishability operators and metrics in the spirit of Theorems 1 and 2. The new results extend the aforementioned results to the new framework and, in addition, allow us to explore also the aforesaid duality relationship when fuzzy (pseudo-)metrics are considered instead of indistinguishability operators.

\section{The New Indistinguishability Operators}

As we have mentioned before, we are interested in proposing a new type of operator that unify the notion of fuzzy (pseudo-)metric and indistinguishability operator in such a way that a unique theoretical basis can be supplied to develop a wide range of applications. To this end, we introduce the notion of modular indistinguishability operator as follows:

Definition 1. Let $X$ be a non-empty set and let $*$ be a t-norm, we will say that fuzzy set $F: X \times X \times] 0, \infty[\rightarrow[0,1]$ is a modular indistinguishability operator for $*$ if for each $x, y, z \in X$ and $t, s>0$ the following axioms are satisfied:

(ME1) $F(x, x, t)=1$;

(ME2) $F(x, y, t)=F(y, x, t)$;

(ME3) $F(x, z, t+s) \geq F(x, y, t) * F(y, z, s)$.

If, in addition, $F$ satisfies for all $x, y \in X$, the following condition:

(ME1') $F(x, y, t)=1$ for all $t>0$ implies $x=y$,

we will say that $F$ separates points.

Moreover, we will say that $F$ is stationary provided that the function $\left.F_{x, y}:\right] 0, \infty[\rightarrow[0,1]$ defined by $F_{x, y}(t)=F(x, y, t)$ is constant for each $x, y \in X$.

Notice that the numerical value $F(x, y, t)$ can understood as the degree up to which $x$ is indistinguishable from $y$ or equivalent to $y$ relative to the value $t$ of the parameter. Moreover, the greater $F(x, y, t)$, the more similar are $x$ and $y$ relative to the value $t$ of the parameter. Clearly, $F(x, y, t)=1$ for all $t>0$ when $x=y$.

It is worth mentioning that the classical notion of indistinguishability operator is recovered when the modular indistinguishability operator $F$ is stationary. In addition, it is clear that a modular indistinguishability operator can be considered as a generalization of the concept of fuzzy (pseudo-)metric. However, there are examples of modular indistinguishability operators that are not a fuzzy (pseudo-)metrics such as the next example shows.

Example 3. Consider a metric $d$ on a non-empty set $X$. Define the fuzzy set $F_{d}$ on $\left.X \times X \times\right] 0, \infty[$ as follows:

$$
F_{d}(x, y, t)= \begin{cases}0, & \text { if } \quad 0<t<d(x, y) \text { and } d(x, y) \neq 0 \\ 1, & \text { if } t \geq d(x, y) \text { and } d(x, y) \neq 0 \\ 1, & \text { if } d(x, y)=0\end{cases}
$$


It is easy to check that $F_{d}$ is a modular indistinguishability operator for the product $t$-norm $*_{P}$. Nevertheless, $\left(F_{d}, *_{P}\right)$ is not a fuzzy (pseudo-)metric because the function $\left.F_{d_{x, y}}:\right] 0, \infty[\rightarrow[0,1]$, defined by $F_{d_{x, y}}(t)=F_{d}(x, y, t)$ is not left-continuous.

The concept of modular indistinguishability operator also generalizes the notion of fuzzy (pseudo-)metric in another outstanding aspect. Observe that, in Definition 1, the continuity on the $t$-norm is not required. Naturally, the assumption of continuity of the $t$-norm is useful from a topological viewpoint, since the continuity is necessary in order to define a topology by means of a family of balls in a similar way like in the pseudo-metric case. However, such an assumption could be limiting the range of applications of such fuzzy measurements in those case where (classical) indistinguishability operators works well. In this direction, modular indistinguishability operators present an advantage with respect to fuzzy (pseudo-)metrics because the involved $t$-norms are not assumed to be continuous.

The following example illustrates the preceding remark providing an instance of modular indistinguishability operator for the Drastic $t$-norm $*_{D}$, which is not a modular indistinguishability operator for any continuous $t$-norm.

Example 4. Let $\varphi$ be the function defined on $] 0, \infty\left[\right.$ by $\varphi(t)=\frac{t}{1+t}$. We define the fuzzy set $F_{D}$ on $[0,1[\times[0,1[\times] 0, \infty[$ as follows:

$$
F_{D}(x, y, t)=\left\{\begin{array}{lll}
1, & \text { for each } t>0, & \text { if } x=y, \\
\max \{x, y, \varphi(t)\}, & \text { for each } t>0, & \text { if } x \neq y .
\end{array}\right.
$$

First of all, note that, for each $x, y \in\left[0,1\left[\right.\right.$ and $t>0$, we have that $F_{D}(x, y, t) \in[0,1[$, since $x, y, \varphi(t) \in\left[0,1\left[\right.\right.$. Thus, $F_{D}$ is a fuzzy set on $[0,1[\times[0,1[\times] 0, \infty[$.

Now, we will see that $F_{D}$ is a modular indistinguishability operator on $\left[0,1\left[\right.\right.$ for $*_{D}$. To this end, let us recall that $*_{D}$ is defined by

$$
a *_{D} b= \begin{cases}0, & \text { if } a, b \in[0,1[ \\ \min \{a, b\}, & \text { elsewhere. }\end{cases}
$$

It is clear that $F_{D}$ satisfies axioms (ME1) and (ME2). Next, we show that $F_{D}$ satisfies (ME3), i.e.,

$$
F_{D}(x, z, t+s) \geq F_{D}(x, y, t) *_{D} F_{D}(y, z, s)
$$

for all $x, y, z \in[0,1[$ and $t, s>0$.

Notice that we can assume that $x \neq z$. Otherwise, the preceding inequality holds trivially. Next, we distinguish two cases:

1. Case 1. $x \neq y$ and $y \neq z$. Then, $F_{D}(x, y, t)=\max \{x, y, \varphi(t)\}<1$ and $F_{D}(y, z, s)=$ $\max \{y, z, \varphi(s)\}<1$, since $x, y, z \in\left[0,1\left[\right.\right.$ and $\varphi(t)<1$ for each $t>0$. Thus, $F_{D}(x, y, t) *_{D}$ $F_{D}(y, z, s)=0$ attending to the definition of $*_{D}$. It follows that $F_{D}(x, z, t+s) \geq F_{D}(x, y, t) *_{D}$ $F_{D}(y, z, s)$.

2. Case 2. $x=y$ or $y=z$ (suppose, without loss of generality, that $x=y$ ). Then, $F_{D}(x, y, t)=1$ and so

$$
F_{D}(x, z, t+s)=F_{D}(y, z, t+s)=\max \{y, z, \varphi(t+s)\} \geq \max \{y, z, \varphi(s)\}=F_{D}(y, z, s),
$$

since $\varphi$ is an increasing function. Thus, $F_{D}(x, z, t+s) \geq F_{D}(y, z, s)=F_{D}(x, y, t) *_{D} F_{D}(y, z, s)$.

Furthermore, the modular indistinguishability operator $F_{D}$ separates points. Indeed, let $x, y \in[0,1[$ and $t>0$. Since $x, y, \varphi(t) \in\left[0,1\left[\right.\right.$ for each $t>0$, we have that, if $x \neq y$, then $F_{D}(x, y, t)=\max \{x, y, \varphi(t)\}<1$. Thus, $F_{D}(x, y, t)=1$ implies $x=y$.

Finally, we will prove that $F_{D}$ is not a modular indistinguishability operator for any continuous t-norm. To this end, we will show that axiom (ME3) is not fulfilled for any t-norm continuous at $(1,1)$. 
Let $*$ be a continuous $t$-norm at $(1,1)$. Then, for each $\epsilon \in] 0,1[$, we can find $\delta \in] 0,1[$ such that $\delta * \delta>$ $1-\epsilon$.

Now, consider $x=0, z=\frac{1}{2}$ and $t=s=1$. Then,

$$
F_{D}(x, z, t+s)=\max \left\{0, \frac{1}{2}, \frac{2}{3}\right\}=\frac{2}{3} .
$$

Taking $\epsilon=\frac{1}{3}$, we can find $\left.\delta \in\right] 0,1\left[\right.$ such that $\delta * \delta>\frac{2}{3}$. Note that, in this case, $\delta>\frac{2}{3}$. Therefore, if we take $y=\delta$, we have that

$$
F_{D}(x, y, t) * F_{D}(y, z, s)=\max \left\{0, y, \frac{1}{2}\right\} * \max \left\{y, \frac{1}{2}, \frac{1}{2}\right\}=y * y>\frac{2}{3}=F_{D}(x, z, t+s) .
$$

Thus, (ME3) is not satisfied.

We end the section with a reflection on axiom (KM1). When such an axiom is considered in the definition of fuzzy (pseudo-)metric (i.e., the fuzzy (pseudo-)metric is considered as a fuzzy set on $X \times X \times[0, \infty[$ instead on $X \times X \times] 0, \infty[$ ), one could wonder whether modular indistinguishability operators would be able to extend the notion of fuzzy (pseudo-)metric in that case. The answer to the posed question is affirmative. In fact, in order to define a new indistinguishability operator for that purpose, we only need to include in the axiomatic in Definition 1 the following axiom:

(ME0) $F(x, y, 0)=0$ for all $x, y \in X$.

Notice that, even in such a case, there exist modular indistinguishability operators that are not fuzzy (pseudo-)metrics. An example of such a kind of operators is given by an easy adaptation of the fuzzy set $F_{d}$ introduced in Example 3. Indeed, we only need to consider such a fuzzy set defined as in the aforesaid example and, in addition, satisfying $F_{d}(x, y, 0)=0$ for all $x, y \in X$. Of course, it is easy to check that $F_{d}$ is a modular indistinguishability operator for the product $t$-norm $*_{P}$, which satisfies (ME0) but $\left(F_{d}, *_{P}\right)$ is not a fuzzy (pseudo-)metric.

\section{The Duality Relationship}

This section is devoted to explore the metric behavior of the new indistinguishability operators. Concretely, we extend, on the one hand, the technique through which a metric can be generated from an indistinguishability operator by means of an additive generator of a $t$-norm (in Section 3.1) and, on the other hand, the technique that allows for inducing an indistinguishability operator from a metric by means of the pseudo-inverse of the additive generator of a $t$-norm (in Section 3.2). The same results are also explored when fuzzy (pseudo-)metrics are considered instead of modular indistinguishability operators.

\subsection{From Modular Indistinguishability Operators to Metrics}

In order to extend Theorem 1 to the modular framework, we need to propose a metric class as a candidate to be induced by a modular indistinguishability operator. We have found that such a candidate is known in the literature as modular metric. Let us recall a few basics about this type of metrics.

According to Chytiakov, V.V. (see [20]), a function $w:] 0, \infty[\times X \times X \rightarrow[0, \infty]$ is a modular metric on a non-empty set $X$ if, for each $x, y, z \in X$ and each $\lambda, \mu>0$, the following axioms are fulfilled:

(MM1) $w(\lambda, x, y)=0$ for all $\lambda>0$ if and only if $x=y$;

(MM2) $w(\lambda, x, y)=w(\lambda, y, x)$;

(MM3) $w(\lambda+\mu, x, z) \leq w(\lambda, x, y)+w(\mu, y, z)$.

If the axiom (MM1) is replaced by the following one, 
(MM1') $w(\lambda, x, x)=0$ for all $\lambda>0$,

then $w$ is a called modular pseudo-metric on $X$.

Of course, the value $w(\lambda, x, y)$ can be understood as a dissimilarity measurement between objects relative to the value $\lambda$ of a parameter. $w_{\lambda}(x, y)$.

Following [20], given $x, y \in X$ and $\lambda>0$, we will denote from now on the value $w(\lambda, x, y)$ by

Notice that, as was pointed out in [20], a (pseudo-)metric is a modular (pseudo-)metric, which is "stationary", i.e., it does not depend on the value $t$ of the parameter. Thus, (pseudo-)metrics on $X$ are modular (pseudo-)metrics $w:] 0, \infty\left[\times X \times X \rightarrow[0, \infty]\right.$ such that the assignment $\left.w_{x, y}:\right] 0, \infty[\rightarrow[0, \infty]$, given by $w_{x, y}(\lambda)=w_{\lambda}(x, y)$ is a constant function for each $x, y \in X$.

The following are well-known examples of modular (pseudo-)metrics.

Example 5. Let $d$ be a (pseudo-)metric on $X$ and let $\varphi:] 0, \infty[\rightarrow] 0, \infty[$ be a non-decreasing function. The functions defined on $] 0, \infty[\times X \times X$ as follows:

(i) $w_{\lambda}^{1}(x, y)= \begin{cases}\infty, & \text { if } x \neq y, \\ 0, & \text { if } x=y,\end{cases}$

(ii) $w_{\lambda}^{2}(x, y)= \begin{cases}\infty, & \text { if } 0<\lambda<d(x, y) \text { and } d(x, y)>0, \\ 0, & \text { if } \lambda \geq d(x, y) \text { and } d(x, y)>0 \\ 0, & \text { if } d(x, y)=0,\end{cases}$

(iii) $w_{\lambda}^{3}(x, y)=\frac{d(x, y)}{\varphi(\lambda)}$,

are modular (pseudo-)metrics on $X$.

Next, we provide an example of modular metric that will be crucial in Section 3.2.

Proposition 1. Let $d$ be a metric space on $X$. Then, the function $w:] 0, \infty[\times X \times X \rightarrow[0, \infty]$ is a modular metric on $X$, where

$$
w_{\lambda}(x, y)=\frac{d^{2}(x, y)}{\lambda}
$$

for each $x, y \in X$ and $\lambda \in] 0, \infty\left[\right.$ (in the last expression, $d^{2}(x, y)$ denotes $(d(x, y))^{2}$, as usual).

Proof. It is clear that axioms (MM1) and (MM2) are satisfied. It remains to show that axiom (MM3) holds. Let $x, y, z \in X$ and $\lambda, \mu \in] 0, \infty[$. Note that

$$
d^{2}(x, z) \leq(d(x, y)+d(y, z))^{2}=d^{2}(x, y)+2 d(x, y) d(y, z)+d^{2}(y, z),
$$

since $d$ is a metric and satisfies the triangle inequality.

From the preceding inequality, we deduce the following one:

$$
\begin{gathered}
\frac{d^{2}(x, y)}{\lambda}+\frac{d^{2}(y, z)}{\mu}-\frac{d^{2}(x, z)}{\lambda+\mu}=\frac{\mu(\lambda+\mu) d^{2}(x, y)+\lambda(\lambda+\mu) d^{2}(y, z)-\lambda \mu d^{2}(x, z)}{\lambda \mu(\lambda+\mu)}= \\
=\frac{\mu \lambda d^{2}(x, y)+\mu^{2} d^{2}(x, y)+\lambda^{2} d^{2}(y, z)+\lambda \mu d^{2}(y, z)-\lambda \mu d^{2}(x, z)}{\lambda \mu(\lambda+\mu)} \geq \\
\geq \frac{\mu \lambda d^{2}(x, y)+\mu^{2} d^{2}(x, y)+\lambda^{2} d^{2}(y, z)+\lambda \mu d^{2}(y, z)-\lambda \mu\left(d^{2}(x, y)+2 d(x, y) d(y, z)+d^{2}(y, z)\right)}{\lambda \mu(\lambda+\mu)}= \\
=\frac{\mu^{2} d^{2}(x, y)+\lambda^{2} d^{2}(y, z)-2 \lambda \mu d(x, y) d(y, z)}{\lambda \mu(\lambda+\mu)}=\frac{(\mu d(x, y)-\lambda d(y, z))^{2}}{\lambda \mu(\lambda+\mu)} \geq 0 .
\end{gathered}
$$


Therefore,

$$
w_{\lambda+\mu}(x, z)=\frac{d^{2}(x, z)}{\lambda+\mu} \leq \frac{d^{2}(x, y)}{\lambda}+\frac{d^{2}(y, z)}{\mu}=w_{\lambda}(x, y)+w_{\mu}(y, z) .
$$

Hence, $w$ satisfies (MM3).

After a brief introduction to modular metric spaces we are able to yield a modular version of Theorem 1.

Theorem 3. Let $X$ be a non-empty set and let $*$ be a continuous t-norm with additive generator $f_{*}:[0,1] \rightarrow[0, \infty]$. If $\diamond$ is a $t$-norm, then the following assertions are equivalent:

(1) $* \leq \diamond$ (i.e., $x * y \leq x \diamond y$ for all $x, y \in[0,1]$ ).

(2) For any modular indistinguishability operator $F$ on $X$ for $\diamond$, the function $\left.\left(w^{F, f_{*}}\right):\right] 0, \infty[\times X \times X \rightarrow[0, \infty]$ defined by

$$
\left(w^{F, f_{*}}\right)_{\lambda}(x, y)=f_{*}(F(x, y, \lambda)),
$$

for each $x, y \in X$ and $\lambda>0$, is a modular pseudo-metric on $X$.

(3) For any modular indistinguishability operator $F$ on $X$ for $\diamond$ that separates points, the function $\left(w^{F, f_{*}}\right)$ : ] $0, \infty[\times X \times X \rightarrow[0, \infty]$ defined by

$$
\left(w^{F, f_{*}}\right)_{\lambda}(x, y)=f_{*}(F(x, y, \lambda)),
$$

for each $x, y \in X$ and $\lambda>0$, is a modular metric on $X$.

Proof. $\quad(1) \Rightarrow(2)$ Suppose that $* \leq \diamond$ and let $F$ be a modular indistinguishability operator on $X$ for $\diamond$. We will see that $\left(w^{F, f_{*}}\right)$ is a modular pseudo-metric on $X$.

(MM1') Let $x \in X$. Since $F(x, x, \lambda)=1$ for each $\lambda>0$, then $\left(w^{F, f_{*}}\right)_{\lambda}(x, x)=f_{*}(F(x, x, \lambda))=$ $f_{*}(1)=0$ for each $\lambda>0$.

(MM2) It is obvious because $F(x, y, \lambda)=F(y, x, \lambda)$ for all $x, y \in X$ and $\lambda>0$.

(MM3) Let $x, y, z \in X$ and $\lambda, \mu>0$. We will show that the following inequality

$$
\left(w^{F, f_{*}}\right)_{\lambda+\mu}(x, z) \leq\left(w^{F, f_{*}}\right)_{\lambda}(x, y)+\left(w^{F, f_{*}}\right)_{\mu}(y, z)
$$

holds. First of all, note that $F$ is also a modular indistinguishability operator for $*$ on $X$ due to $\diamond \geq *$. Then, the following inequality is satisfied:

$$
F(x, z, \lambda+\mu) \geq F(x, y, \lambda) * F(y, z, \mu)=f_{*}^{(-1)}\left(f_{*}(F(x, y, \lambda))+f_{*}(F(y, z, \mu))\right) .
$$

Taking into account that $f_{*}$ is an additive generator, and thus a decreasing function, we have that

$$
f_{*}(F(x, z, \lambda+\mu)) \leq f_{*}\left(f_{*}^{(-1)}\left(f_{*}(F(x, y, \lambda))+f_{*}(F(y, z, \mu))\right)\right) .
$$

Now, we will distinguish two different cases:

(a) Suppose that $f_{*}(F(x, y, \lambda))+f_{*}(F(y, z, \mu)) \in \operatorname{Ran}\left(f_{*}\right)$.

Since $f_{*}$ is an additive generator of the $t$-norm $*$, we have that $\left.f_{*} \circ f_{*}^{(-1)}\right|_{\operatorname{Ran}\left(f_{*}\right)}=\left.i d\right|_{\operatorname{Ran}\left(f_{*}\right)}$. Then,

$$
f_{*}\left(f_{*}^{(-1)}\left(f_{*}(F(x, y, \lambda))+f_{*}(F(y, z, \mu))\right)\right)=f_{*}(F(x, y, \lambda))+f_{*}(F(y, z, \mu)) .
$$


It follows that

$$
\begin{aligned}
\left(w^{F, f_{*}}\right)_{\lambda+\mu}(x, z)= & f_{*}(F(x, z, \lambda+\mu)) \leq f_{*}(F(x, y, \lambda))+f_{*}(F(y, z, \mu))= \\
& =\left(w^{F, f_{*}}\right)_{\lambda}(x, y)+\left(w^{F, f_{*}}\right)_{\mu}(y, z) .
\end{aligned}
$$

(b) Suppose that $f_{*}(F(x, y, \lambda))+f_{*}(F(y, z, \mu)) \notin \operatorname{Ran}\left(f_{*}\right)$. Since $f_{*}$ is an additive generator of the $t$-norm $*$, we have that $f_{*}(a)+f_{*}(b) \in \operatorname{Ran}\left(f_{*}\right) \cup\left[f_{*}(0), \infty\right]$ for each $a, b \in[0,1]$. Then,

$$
f_{*}(F(x, y, \lambda))+f_{*}(F(y, z, \mu))>f_{*}(0) .
$$

Thus, we obtain

$$
f_{*}(F(x, z, \lambda+\mu)) \leq f_{*}(0)<f_{*}(F(x, y, \lambda))+f_{*}(F(y, z, \mu))
$$

where we have that

$$
\left(w^{F, f_{*}}\right)_{\lambda+\mu}(x, z) \leq\left(w^{F, f_{*}}\right)_{\lambda}(x, y)+\left(w^{F, f_{*}}\right)_{\mu}(y, z),
$$

as we claimed.

Therefore, $\left(w^{F, f_{*}}\right)$ is a modular pseudo-metric on $X$.

$(2) \Rightarrow(3)$ Let $F$ be a modular indistinguishability operator on $X$ for $\diamond$ that separates points. By our assumption, $\left(w^{F, f_{*}}\right)$ is a pseudo-modular metric on $X$. We will see that $\left(w^{F, f_{*}}\right)$ is a modular metric on $X$.

Let $x, y \in X$ such that $\left(w^{F, f_{*}}\right)_{\lambda}(x, y)=0$ for all $\lambda>0$. By definition, we have that $f_{*}(F(x, y, \lambda))=0$ for all $\lambda>0$. Then, $F(x, y, \lambda)=1$ for all $\lambda>0$, since $f_{*}$ is an additive generator of $*$. Therefore, $x=y$, since $F$ is a modular indistinguishability operator on $X$ for $\diamond$ that separates points.

$(3) \Rightarrow(1)$ Suppose that, for any modular indistinguishability operator $F$ on $X$ for $\diamond$ that separates points, the function $\left(w^{F, f_{*}}\right)$ is a modular metric on $X$. We will show that $\diamond \geq *$. To this end, we will prove that $a \diamond b \geq a * b$ provided $a, b \in[0,1[$. Note that the preceding inequality is obvious whenever either $a=1$ or $b=1$.

Let $a, b \in[0,1[$. Consider a set constituted by three distinct points $X=\{x, y, z\}$. We define a fuzzy set $F$ on $X \times X \times] 0, \infty[$ as follows:

$$
F(u, v, t)=F(v, u, t)= \begin{cases}1, & \text { if } u=v \\ a \diamond b, & \text { if } u=x \text { and } v=z \\ a, & \text { if } u=x \text { and } v=y \\ b, & \text { if } u=y \text { and } v=z\end{cases}
$$

for all $t>0$.

It is easy to verify, attending to its definition, that $F$ is a modular indistinguishability operator on $X$ for $\diamond$ that separates points. Thus, $\left(w^{F, f_{*}}\right)$ is a modular metric on $X$. Therefore, given $\lambda>0$, we have that

$$
f_{*}(a \diamond b)=\left(w^{F, f_{*}}\right)_{2 \lambda}(x, z) \leq\left(w^{F, f_{*}}\right)_{\lambda}(x, y)+\left(w^{F, f_{*}}\right)_{\lambda}(y, z)=f_{*}(a)+f_{*}(b) .
$$

Notice that, for each $c \in[0,1]$, we have that $\left(f_{*}^{(-1)} \circ f_{*}\right)(c)=c, a * b=f_{*}^{(-1)}\left(f_{*}(a)+f_{*}(b)\right)$ and that $f_{*}^{(-1)}$ is decreasing, since $f_{*}$ is an additive generator of the $t$-norm $*$. Taking into account the preceding facts and, from the above inequality, we deduce that

$$
a \diamond b=f_{*}^{(-1)}\left(f_{*}(a \diamond b)\right) \geq f_{*}^{(-1)}\left(f_{*}(a)+f_{*}(b)\right)=a * b,
$$


as we claimed.

This last implication concludes the proof.

In order to illustrate the technique introduced in the above theorem, we provide two corollaries that establish the particular cases for the Luckasievicz $t$-norm and the usual product. With this aim, we recall that an additive generator $f_{*_{L}}$ of $*_{L}$ and $f_{*_{P}}$ of $*_{P}$ is given by

$$
\begin{aligned}
& f_{*_{L}}(a)=1-a, \\
& f_{*_{P}}(a)=-\log (a),
\end{aligned}
$$

for each $a \in[0,1]$, respectively. Of course, we have adopted the convention that $\log (0)=-\infty$.

Corollary 1. Let $X$ be a non-empty set. If $\diamond$ is a $t$-norm, then the following assertions are equivalent:

(1) $*_{L} \leq \diamond$.

(2) For any modular indistinguishability operator F on $X$ for $\diamond$, the function $\left.\left(w^{F, f_{*_{L}}}\right):\right] 0, \infty[\times X \times X \rightarrow[0, \infty]$ defined by

$$
\left(w^{F, f_{*}}\right)_{\lambda}(x, y)=1-F(x, y, \lambda),
$$

for each $x, y \in X$ and $\lambda>0$, is a modular pseudo-metric on $X$.

(3) For any modular indistinguishability operator $F$ on $X$ for $\diamond$ that separates points, the function $\left.\left(w^{F, f_{*}}\right):\right] 0, \infty[\times X \times X \rightarrow[0, \infty]$ defined by

$$
\left(w^{F, f_{*}}\right)_{\lambda}(x, y)=1-F(x, y, \lambda),
$$

for each $x, y \in X$ and $\lambda>0$, is a modular metric on $X$.

Corollary 2. Let $X$ be a non-empty set. If $\diamond$ is a $t$-norm, then the following assertions are equivalent:

(1) $*_{p} \leq \diamond$.

(2) For any modular indistinguishability operator $F$ on $X$ for $\diamond$, the function $\left.\left(w^{F, f_{* P}}\right):\right] 0, \infty[\times X \times X \rightarrow[0, \infty]$ defined by

$$
\left(w^{F, f_{*_{P}}}\right)_{\lambda}(x, y)=-\log (F(x, y, \lambda)),
$$

for each $x, y \in X$ and $\lambda>0$, is a modular pseudo-metric on $X$.

(3) For any modular indistinguishability operator $F$ on $X$ for $\diamond$ that separates points, the function $\left.\left(w^{F, f_{* P}}\right):\right] 0, \infty[\times X \times X \rightarrow[0, \infty]$ defined by

$$
\left(w^{F, f_{*_{P}}}\right)_{\lambda}(x, y)=-\log (F(x, y, \lambda)),
$$

for each $x, y \in X$ and $\lambda>0$, is a modular metric on $X$.

Theorem 3 also gives a specific method to generate modular metrics when we focus our attention on fuzzy (pseudo-)metrics instead of modular indistinguishability operators in general.

Corollary 3. Let $X$ be a non-empty set and let $*$ be a t-norm with additive generator $f_{*}:[0,1] \rightarrow[0, \infty]$. If $\diamond$ is a continuous $t$-norm, then the following assertions are equivalent:

(1) $* \leq \diamond$.

(2) For any fuzzy pseudo-metric $(M, \diamond)$ on $X$, the function $\left.\left(w^{M, f_{*}}\right):\right] 0, \infty[\times X \times X \rightarrow[0, \infty]$ defined by

$$
\left(w^{M, f_{*}}\right)_{\lambda}(x, y)=f_{*}(M(x, y, \lambda)),
$$

for each $x, y \in X$ and $\lambda>0$, is a modular pseudo-metric on $X$. 
(3) For any fuzzy metric $(M, \diamond)$ on $X$, the function $\left.\left(w^{M, f_{*}}\right):\right] 0, \infty[\times X \times X \rightarrow[0, \infty]$ defined by

$$
\left(w^{M, f_{*}}\right)_{\lambda}(x, y)=f_{*}(M(x, y, \lambda))
$$

for each $x, y \in X$ and $\lambda>0$, is a modular metric on $X$.

As a consequence of the preceding result, we obtain immediately the following one.

Corollary 4. Let $X$ be a non-empty set and let $*$ be a continuous $t$-norm with additive generator $f_{*}:[0,1] \rightarrow[0, \infty]$. Then, the following assertions are equivalent:

(1) For any fuzzy pseudo-metric $(M, *)$ on $X$, the function $\left.\left(w^{M, f_{*}}\right):\right] 0, \infty[\times X \times X \rightarrow[0, \infty]$ defined by

$$
\left(w^{M, f_{*}}\right)_{\lambda}(x, y)=f_{*}(M(x, y, \lambda))
$$

for each $x, y \in X$ and $\lambda>0$, is a modular pseudo-metric on $X$.

(2) For any fuzzy metric $(M, *)$ on $X$, the function $\left.\left(w^{M, f_{*}}\right):\right] 0, \infty[\times X \times X \rightarrow[0, \infty]$ defined by

$$
\left(w^{M, f_{*}}\right)_{\lambda}(x, y)=f_{*}(M(x, y, \lambda))
$$

for each $x, y \in X$ and $\lambda>0$, is a modular metric on $X$.

It is clear that, when we consider stationary modular indistinguishability operators in the statement of Theorem 3, we obtain as a particular case Theorem 1 and, thus, the classical technique to induce a metric from an indistinguishability operator by means of an additive generator. Clearly, if we replace modular indistinguishability operators by stationary fuzzy metrics, we obtain a more restrictive version of the classical technique, provided by Theorem 3 , because it only remains valid for continuous $t$-norms.

\subsection{From Modular (Pseudo-)Metrics to Modular Indistinguishability Operators}

As was mentioned above, the main goal of this subsection is to provide a version of Theorem 2 when we consider a modular (pseudo-)metric instead of a (pseudo-)metric. Thus, we give a technique to induce a modular indistinguishability operator from a modular (pseudo-)metric by means of the pseudo-inverse of the additive generator of a t-norm. To this end, let us recall the following representation result, which will be crucial in our subsequent discussion, holds for continuous $t$-norms:

Theorem 4. A binary operator $*$ in $[0,1]$ is a continuous Archimedean $t$-norm if and only if there exists a continuous additive generator $f_{*}$ such that

$$
x * y=f_{*}^{(-1)}\left(f_{*}(x)+f_{*}(y)\right),
$$

where the pseudo-inverse $f_{*}^{(-1)}$ is given by

$$
f_{*}^{(-1)}(y)=f^{-1}\left(\min \left\{f_{*}(0), y\right\}\right)
$$

for all $y \in[0, \infty]$

In the next result, we introduce the promised technique.

Theorem 5. Let $*$ be a continuous $t$-norm with additive generator $f_{*}:[0,1] \rightarrow[0, \infty]$. If $w$ is a modular pseudo-metric on $X$, then the function $\left.F^{w, f_{*}}: X \times X \times\right] 0, \infty[\rightarrow[0,1]$ defined, for all $x, y \in X$ and $t>0$, by

$$
F^{w, f_{*}}(x, y, t)=f_{*}^{(-1)}\left(w_{t}(x, y)\right)
$$


is a modular indistinguishability operator for $*$. Moreover, the modular indistinguishability operator $F^{w,} f_{*}$ separates points if and only if $w$ is a modular metric on $X$.

Proof. Let $*$ be a continuous Archimedean $t$-norm with additive generator $f_{*}:[0,1] \rightarrow[0, \infty]$ and consider $w$ a modular pseudo-metric on $X$.

We define the function $\left.F^{w, f_{*}}: X \times X \times\right] 0, \infty[\rightarrow[0,1]$ as follows:

$$
F^{w, f_{*}}(x, y, t)=f_{*}^{(-1)}\left(w_{t}(x, y)\right)
$$

for all $x, y \in X$ and $t>0$. We will see that $F^{w, f_{*}}$ is a modular inidistinguishability operator for $*$.

(ME1) Let $x \in X$. Since $w$ is a modular pseudo-metric on $X$, we have that $w_{t}(x, x)=0$ for all $t>0$.

Therefore, $F^{w, f_{*}}(x, x, t)=f_{*}^{(-1)}\left(w_{t}(x, x)\right)=f_{*}^{(-1)}(0)=1$ for all $t>0$.

(ME2) Is a consequence of the definition of $F^{w, f_{*}}$, since $w$ is a modular pseudo-metric and so it satisfies that $w_{t}(x, y)=w_{t}(y, x)$ for each $x, y \in X$ and $t>0$.

(ME3) Let $x, y, z \in X$ and $t, s>0$. On the one hand, by expression (2), we deduce that

$$
F^{w, f_{*}}(x, z, t+s)=f_{*}^{(-1)}\left(w_{t+s}(x, z)\right)=f_{*}^{-1}\left(\min \left\{f_{*}(0), w_{t+s}(x, z)\right\}\right) .
$$

Now, since $w$ is a modular pseudo-metric on $X$, then

$$
w_{t+s}(x, z) \leq w_{t}(x, y)+w_{s}(y, z)
$$

and, hence,

$$
F^{w, f_{*}}(x, z, t+s) \geq f_{*}^{-1}\left(\min \left\{f_{*}(0), w_{t}(x, y)+w_{s}(y, z)\right\}\right) .
$$

On the other hand, we have that

$$
\begin{gathered}
F^{w, f_{*}}(x, y, t) * F^{w, f_{*}}(y, z, s)=f_{*}^{(-1)}\left(f_{*}\left(F^{w, f_{*}}(x, y, t)\right)+f_{*}\left(F^{w, f_{*}}(y, z, s)\right)\right)= \\
=f_{*}^{-1}\left(\min \left\{f_{*}(0), f_{*}\left(F^{w, f_{*}}(x, y, t)\right)+f_{*}\left(F^{w, f_{*}}(y, z, s)\right)\right\}\right) .
\end{gathered}
$$

Moreover, by expression (2), we obtain that

$$
f_{*}\left(F^{w, f_{*}}(x, y, t)\right)=f_{*}\left(f_{*}^{(-1)}\left(w_{t}(x, y)\right)\right)=\min \left\{f_{*}(0), w_{t}(x, y)\right\}
$$

and

$$
f_{*}\left(F^{w, f_{*}}(y, z, s)\right)=f_{*}\left(f_{*}^{(-1)}\left(w_{s}(y, z)\right)\right)=\min \left\{f_{*}(0), w_{s}(y, z)\right\} .
$$

To finish the proof, we will see that

$$
\min \left\{f_{*}(0), w_{t}(x, y)+w_{s}(y, z)\right\}=\min \left\{f_{*}(0), \min \left\{f_{*}(0), w_{t}(x, y)\right\}+\min \left\{f_{*}(0), w_{s}(y, z)\right\}\right\} .
$$

To this end, we will distinguish three cases:

Case 1. $f_{*}(0) \leq w_{t}(x, y)$ and $f_{*}(0) \leq w_{s}(y, z)$. Then, we have that

$$
\min \left\{f_{*}(0), w_{t}(x, y)+w_{s}(y, z)\right\}=f_{*}(0)
$$

and

$$
\min \left\{f_{*}(0), \min \left\{f_{*}(0), w_{t}(x, y)\right\}+\min \left\{f_{*}(0), w_{s}(y, z)\right\}\right\}=\min \left\{f_{*}(0), f_{*}(0)+f_{*}(0)\right\}=f_{*}(0) .
$$


Case 2. $f_{*}(0)>w_{t}(x, y)$ and $f_{*}(0) \leq w_{\mathcal{S}}(y, z)$ (the case $f_{*}(0) \leq w_{t}(x, y)$ and $f_{*}(0)>w_{\mathcal{S}}(y, z)$ runs following the same arguments). It follows that

$$
\min \left\{f_{*}(0), w_{t}(x, y)+w_{s}(y, z)\right\}=f_{*}(0)
$$

and

$$
\min \left\{f_{*}(0), \min \left\{f_{*}(0), w_{t}(x, y)\right\}+\min \left\{f_{*}(0), w_{s}(y, z)\right\}\right\}=\min \left\{f_{*}(0), w_{t}(x, y)+f_{*}(0)\right\}=f_{*}(0) .
$$

Case 3. $f_{*}(0)>w_{t}(x, y)$ and $f_{*}(0)>w_{\mathcal{S}}(y, z)$. Then, we have that

$$
\min \left\{f_{*}(0), \min \left\{f_{*}(0), w_{t}(x, y)\right\}+\min \left\{f_{*}(0), w_{\mathcal{S}}(y, z)\right\}\right\}=\min \left\{f_{*}(0), w_{t}(x, y)+w_{\mathcal{S}}(y, z)\right\} .
$$

Therefore,

$$
\begin{aligned}
& F^{w, f_{*}}(x, z, t+s) \geq f_{*}^{-1}\left(\min \left\{f_{*}(0), f_{*}\left(F^{w, f_{*}}(x, y, t)\right)+f_{*}\left(F^{w, f_{*}}(y, z, s)\right)\right\}\right) \\
&=F^{w, f_{*}}(x, y, t) * F^{w, f_{*}}(y, z, s),
\end{aligned}
$$

whence we deduce that $F^{w, f_{*}}$ is a modular indistinguishability operator for $*$ on $X$.

Finally, it is clear that $F^{w, f_{*}}(x, y, t)=1$ for all $x, y \in X$ and $t>0$ if, and only if, $f_{*}^{(-1)}\left(w_{t}(x, y)\right)=1$ for all $x, y \in X$ and $t>0$. Since $f_{*}^{(-1)}\left(w_{t}(x, y)\right)=1$ for all $x, y \in X$ and $t>0$ if, and only if, $w_{t}(x, y)=0$ for all $x, y \in X$ and $t>0$, we immediately obtain that $F^{w, f_{*}}$ is a modular indistinguishability operator that separates points if, and only if, $w$ is a modular metric on $X$.

Next, we specify the method given in Theorem 5 for the $t$-norms $*_{L}$ and $*_{P}$. Note that the pseudo-inverse of the additive generator $f_{*_{L}}$ and $f_{*_{P}}$ is given by

$$
f_{*_{L}}^{(-1)}(b)= \begin{cases}1-b, & \text { if } b \in[0,1[ \\ 0, & \text { if } b \in[1, \infty]\end{cases}
$$

and

$$
f_{* P}^{(-1)}(b)=\mathrm{e}^{-b},
$$

for each $b \in[0, \infty]$, respectively, where we have adopted the convention that $\mathrm{e}^{-\infty}=0$.

Corollary 5. If $w$ is a modular pseudo-metric on $X$, then the function $\left.F^{w, f_{*_{L}}}: X \times X \times\right] 0, \infty[\rightarrow[0,1]$ defined, for all $x, y \in X$ and $t>0$, by

$$
F^{w, f_{*}}(x, y, t)= \begin{cases}1-w_{t}(x, y), & \text { if } w_{t}(x, y) \in[0,1[, \\ 0, & \text { if } w_{t}(x, y) \in[1, \infty],\end{cases}
$$

is a modular indistinguishability operator for $*_{L}$. Moreover, the modular indistinguishability operator $F^{w, f_{*}}$ separates points if and only if $w$ is a modular metric on $X$.

Corollary 6. If $w$ is a modular pseudo-metric on $X$, then the function $\left.F^{w, f_{*}}: X \times X \times\right] 0, \infty[\rightarrow[0,1]$ defined, for all $x, y \in X$ and $t>0$, by

$$
F^{w, f_{*}}(x, y, t)=\mathrm{e}^{-w_{t}(x, y)},
$$

is a modular indistinguishability operator for $*_{P}$. Moreover, the modular indistinguishability operator $F^{w, f_{*_{L}}}$ separates points if and only if $w$ is a modular metric on $X$. 
In the light of Theorem 5, it seems natural to ask if the continuity of the $t$-norm can be eliminated from the assumptions of such a result. The next example gives a negative answer to that question. In particular, it proves that there are fuzzy sets $F^{w, f_{*}}$, given by Theorem 5 , that are not modular indistinguishability operators when the $t$-norm $*$ under consideration is not continuous.

Example 6. Consider the Euclidean metric $d_{E}$ on $\mathbb{R}$. By Proposition 1, the function $w^{E}$ is a modular metric on $\mathbb{R}$, where

$$
w_{\lambda}^{E}(x, y)=\frac{\left(d_{E}(x, y)\right)^{2}}{\lambda}
$$

for all $x, y \in \mathbb{R}$ and $\lambda>0$. Consider the additive generator $f_{*_{D}}$ of the non-continuous $t$-norm $*_{D}$. Recall that $f_{*_{D}}$ is given by

$$
f_{*_{D}}(x)= \begin{cases}0, & \text { if } x=1, \\ 2-x, & \text { if } x \in[0,1[.\end{cases}
$$

An easy computation shows that its pseudo-inverse is given by

$$
f_{*_{D}}^{(-1)}(x)= \begin{cases}1, & \text { if } x \in[0,1], \\ 2-x, & \text { if } x \in] 1,2], \\ 0, & \text { if } x \in] 2, \infty[.\end{cases}
$$

Next, we show that we can find $x, y, z \in \mathbb{R}$ and $\lambda, \mu \in] 0, \infty[$ such that

$$
F^{w^{E}, f_{*_{D}}}(x, z, \lambda+\mu)<F^{w^{E}, f_{*_{D}}}(x, y, \lambda) *_{D} F^{w^{E}, f_{*_{D}}}(y, z, \mu) .
$$

Let $x=0, y=1$ and $z=2$, and consider $\lambda=\mu=1$. Then,

$$
\begin{gathered}
w_{\lambda+\mu}^{E}(x, z, \lambda)=\frac{\left(d_{E}(x, z)\right)^{2}}{\lambda+\mu}=\frac{2^{2}}{2}=2, \\
w_{\lambda}^{E}(x, y)=\frac{\left(d_{E}(x, y)\right)^{2}}{\lambda}=\frac{1^{2}}{1}=1,
\end{gathered}
$$

and

$$
w_{\mu}^{E}(y, z)=\frac{\left(d_{E}(y, z)\right)^{2}}{\mu}=\frac{1^{2}}{1}=1
$$

Therefore,

$$
0=f_{*_{D}}^{(-1)}(2)=F^{w^{E}, f_{*_{D}}}(x, z, \lambda+\mu)<F^{w^{E}, f_{*_{D}}}(x, y, \lambda) *_{D} F^{w^{E}, f_{*_{D}}}(y, z, \mu)=f_{*_{D}}^{(-1)}(1) *_{D} f_{*_{D}}^{(-1)}(1)=1 .
$$

Since the continuity is a necessary hypothesis in the statement of Theorem 5, one could expect that the following result would be true.

"Let $*$ be a continuous Archimedean $t$-norm with additive generator $f_{*}:[0,1] \rightarrow[0, \infty]$. If $w$ is a modular pseudo-metric on $X$, then the pair $\left(M^{w, f_{*}}, *\right)$ is a fuzzy (pseudo-)metric, where the fuzzy set $\left.M^{w, f_{*}}: X \times X \times\right] 0, \infty[$ is given, for all $x, y \in X$ and $t>0$, by

$$
M^{w, f_{*}}(x, y, t)=f_{*}^{(-1)}\left(w_{t}(x, y)\right)
$$

Moreover, $\left(M^{w, f_{*}}, *\right)$ is a fuzzy metric if and only if $w$ is a modular metric on $X . "$

Nevertheless, the following example proves that such a result does not hold. In fact, the technique provided by Theorem 5 does not give in general a fuzzy (pseudo-)metric. 
Example 7. Let $d$ be a metric on a non-empty set X. Consider the modular metric $w^{2}$ on $X$ introduced in Example 5, that is,

$$
w_{t}^{2}(x, y)= \begin{cases}\infty, & \text { if } \quad 0<t<d(x, y) \text { and } d(x, y)>0 \\ 0, & \text { if } t \geq d(x, y) \text { and } d(x, y)>0 \\ 0, & \text { if } d(x, y)=0\end{cases}
$$

for all $x, y \in X$ and $t>0$. Then, it is not hard to check that the pair $\left(M^{w^{2}, f_{*_{P}}, *_{P}}\right)$ is not a fuzzy (pseudo-)metric, where the fuzzy set $M^{w^{2}, f_{* P}}$ is given by

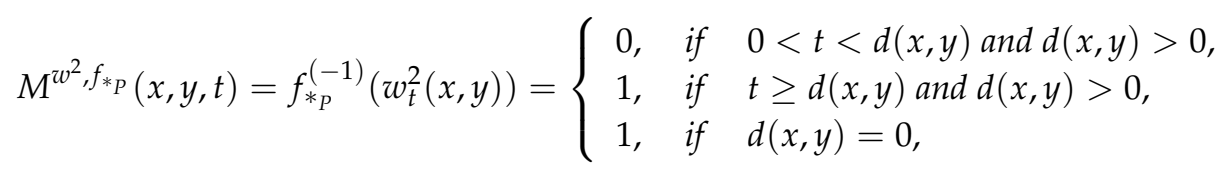

for all $x, y \in X$ and $t>0$. Notice that $\left(M^{w^{2}, f_{* P}, *_{P}}\right)$ fails to fulfill axiom (KM5), i.e., the function $M_{x, y}^{w^{2}, f_{*_{P}}}$ : ] $0, \infty[\rightarrow[0,1]$ is not left-continuous.

The preceding example suggests the study of those conditions that a modular (pseudo-)metric must satisfy in order to induce a fuzzy (pseudo-) metric by means of the technique exposed in Theorem 5. The following lemma, whose proof was given in [20], will help us to find it.

Lemma 1. Let $w$ be a modular (pseudo-)metric on $X$. Then, for each $x, y \in X$, we have that $w_{s}(x, y) \geq w_{t}(x, y)$ whenever $s, t \in] 0, \infty[$ with $s<t$.

Taking into account the preceding lemma, the next result provides a condition that is useful for our target.

Proposition 2. Let $w$ be a modular pseudo-metric on $X$. The function $\tilde{w}:] 0, \infty[\times X \times X \rightarrow[0, \infty]$ given, for each $x, y \in X$ and $t>0$, by

$$
\tilde{w}_{\lambda}(x, y)=\inf _{0<t<\lambda} w_{t}(x, y)
$$

is a modular pseudo-metric on $X$ such that for each $x, y \in X$ the function $\left.\tilde{w}_{x, y}:\right] 0, \infty[\rightarrow] 0, \infty[$ is left continuous, where $\tilde{w}_{x, y}(\lambda)=\tilde{w}_{\lambda}(x, y)$ for each $\left.\lambda \in\right] 0, \infty[$. Furthermore, $\tilde{w}$ is a modular metric on $X$ if and only if $w$ it is so.

Proof. It is obvious that $\tilde{w}$ satisfies axiom (MM2). Next, we show that $\tilde{w}$ satisfies axioms (MM1') and (MM3).

(MM1') Fix $x \in X$ and let $\lambda \in] 0, \infty\left[\right.$. Since $w$ is a modular pseudo-metric on $X$, then $w_{t}(x, x)=0$ for each $t>0$. Therefore,

$$
\tilde{w}_{\lambda}(x, x)=\inf _{0<t<\lambda} w_{t}(x, x)=0 .
$$

(MM3) Let $x, y, z \in X$ and $\lambda, \mu \in] 0, \infty[$. Next, we prove that

$$
\tilde{w}_{\lambda+\mu}(x, z) \leq \tilde{w}_{\lambda}(x, y)+\tilde{w}_{\mu}(y, z)
$$

With this aim, note that, given $u, v \in X$ and $\alpha \in] 0, \infty[$, we have that, for each $\epsilon \in] 0, \infty[$, we can find $t \in] 0, \alpha\left[\right.$ satisfying $w_{t}(u, v)<\tilde{w}_{\alpha}(u, v)+\epsilon$.

Fixing an arbitrary $\epsilon \in] 0, \infty[$, we can then find $t \in] 0, \lambda[$ and $s \in] 0, \mu\left[\right.$ such that $w_{t}(x, y)<$ $\tilde{w}_{\lambda}(x, y)+\epsilon / 2$ and $w_{s}(y, z)<\tilde{w}_{\mu}(y, z)+\epsilon / 2$. Therefore,

$$
\tilde{w}_{\lambda+\mu}(x, z) \leq w_{t+s}(x, z) \leq w_{t}(x, y)+w_{s}(y, z)<\tilde{w}_{\lambda}(x, y)+\tilde{w}_{\mu}(y, z)+\epsilon,
$$


since $w$ is a pseudo-metric on $X$. Taking into account that $\epsilon \in] 0, \infty[$ is arbitrary, we conclude that

$$
\tilde{w}_{\lambda+\mu}(x, z) \leq \tilde{w}_{\lambda}(x, y)+\tilde{w}_{\mu}(y, z)
$$

Thus, $\tilde{w}$ is a modular pseudo-metric on $X$.

We will continue showing that, for each $x, y \in X$, the function $\left.\tilde{w}_{x, y}:\right] 0, \infty[\rightarrow] 0, \infty$ [ is left continuous. Fix $x, y \in X$ and consider an arbitrary $\left.\lambda_{0} \in\right] 0, \infty[$. Then, given $\epsilon \in] 0, \infty[$, we can find $\delta \in] 0, \infty[$ such that

$$
\tilde{w}_{\lambda}(x, y)-\tilde{w}_{\lambda_{0}}(x, y)<\epsilon
$$

for each $\left.\lambda \in] \lambda_{0}-\delta, \lambda_{0}\right]$ (note that $\tilde{w}_{\lambda}(x, y) \geq \tilde{w}_{\lambda_{0}}(x, y)$ for each $\left.\left.\lambda \in\right] \lambda_{0}-\delta, \lambda_{0}\right]$ by Lemma 1 ). Indeed, let $\epsilon \in] 0, \infty[$. As before, we can find $t \in] 0, \lambda_{0}[$ such that

$$
w_{t}(x, y)<\tilde{w}_{\lambda_{0}}(x, y)+\epsilon
$$

and, again by Lemma 1 , we have that $w_{s}(x, y)<\tilde{w}_{\lambda_{0}}(x, y)+\epsilon$ for each $\left.\left.s \in\right] t, \lambda_{0}\right]$. Therefore, taking $\delta=\lambda_{0}-t$, we have that

$$
\tilde{w}_{\lambda}(x, y)-\tilde{w}_{\lambda_{0}}(x, y) \leq w_{\lambda}(x, y)-\tilde{w}_{\lambda_{0}}(x, y)<\epsilon,
$$

for each $\left.\lambda \in] \lambda_{0}-\delta, \lambda_{0}\right]$, as we claimed. Thus, $\tilde{w}_{x, y}$ is left-continuous on $] 0, \infty\left[\right.$ since $\lambda_{0}$ is arbitrary.

Finally, it is easy to verify that $\tilde{w}$ is a modular metric on $X$, if and only if $w$, it is so. Indeed, $\tilde{w}$ is a modular metric on $X$ if and only if $\tilde{w}_{\lambda}(x, y)=0$ for each $\left.\lambda \in\right] 0, \infty\left[\right.$ implies $x=y$, but $\tilde{w}_{\lambda}(x, y)=$ $\inf _{0<t<\lambda} w_{t}(x, y)=0$ for each $\left.\lambda \in\right] 0, \infty\left[\right.$ if and only if $w_{t}(x, y)=0$ for each $\left.t \in\right] 0, \infty[$, which concludes the proof.

Observe that, in the preceding result, $\tilde{w}$ coincides with $w$, whenever $w_{x, y}$ is a left-continuous function, for each $x, y \in X$.

Proposition 2 and Theorem 5 allow us to give the searched method for constructing a fuzzy pseudo-metric from a modular pseudo-metric.

Theorem 6. Let $*$ be a continuous $t$-norm with additive generator $f_{*}:[0,1] \rightarrow[0, \infty]$. If $w$ is a modular pseudo-metric on $X$, then the pair $\left(M^{w, f_{*}}, *\right)$ is a fuzzy pseudo-metric on $X$, where the fuzzy set $M^{w, f_{*}}$ : $X \times X \times[0, \infty[$ is defined, for all $x, y \in X$, by

$$
M^{w, f_{*}}(x, y, t)=f_{*}^{(-1)}\left(\tilde{w}_{t}(x, y)\right),
$$

where $\tilde{w}_{t}(x, y)=\inf _{0<\lambda<t} w_{\lambda}(x, y)$. Moreover, $\left(M^{w, f_{*}}, *\right)$ is a fuzzy metric on $X$ if and only if $w$ is a modular metric on $X$.

Proof. By Proposition 2, we deduce that $\tilde{w}_{x, y}$ is a modular pseudo-metric on $X$. Theorem 5 guarantees that $M^{w}, f_{*}$ is a modular indistinguishability operator for $*$ on $X$. Moreover, continuity of $f_{*}^{(-1)}$ and the left-continuity, provided by Proposition 2, of the function $\tilde{w}_{x, y}$ guarantee that axiom (KM5) is fulfilled. Thus, the pair $\left(M^{w, f_{*}}, *\right)$ is a fuzzy pseudo-metric on $X$. Finally, by Proposition 2 and Theorem 5 , it is obvious that $\left(M^{w, f_{*}}, *\right)$ is a fuzzy metric on $X$ if and only if $w$ is a modular metric on $X$.

\section{Discussion}

In the literature, there are two tools that allow for measuring the degree of similarity between objects. They are the so-called indistinguishability operators and fuzzy metrics. The former provide the degree up to which two objects are equivalent when there is a limitation on the accuracy of measurement between the objects being compared. The fuzzy metrics provide the degree up to which two objects are equivalent when the measurement is relative to a parameter. Motivated by the fact 
that none of these types of similarity measurements generalizes the other, we have introduced a new notion of indistinguishability operator that unifies both notions, fuzzy metric and indistinguishability operator under a new one. Moreover, we have explored the metric behavior of this new kind of operator in such a way that the new results extend the classical results to the new framework and, in addition, allow for exploring the aforesaid duality relationship when fuzzy metrics are considered instead of indistinguishability operators. The fact that the new notion of indistinguishability operator does not involve the continuity on the $t$-norm in their axiomatic presents an advantage with respect to the fuzzy metrics. The assumption of continuity could be limiting the range of applications of fuzzy metrics in those cases where (classical) indistinguishability operators work well. A future work remains open to study which properties of classical indistinguishability operators are also verified in the new framework. In addition, the utility of the new operators in applied problems must be explored.

\section{Conclusions}

In the present paper we have introduced a new type of indistinguishability operators which generalize the notion of fuzzy metric and the classical notion of indistinguishability operator. Moreover, we have discussed the relationship between the new operators and metrics. Specifically, we have proved the existence of a duality between them and the so-called modular metrics, which provide a dissimilarity measurement between objects relative to a parameter. Furthermore, we have yielded examples that illustrate the new results.

Acknowledgments: Oscar Valero acknowledges the support of the Spanish Ministry of Economy and Competitiveness under Grant TIN2016-81731-REDT (LODISCO II) and Agencia Estatal de Investigación (AEI)/Fondo Europeo de Desarrollo Regional (FEDER), UE funds.

Author Contributions: Juan-José Miñana and Oscar Valero have contributed equally to this work.

Conflicts of Interest: The authors declare no conflict of interest.

\section{References}

1. Trillas, E. Assaig sobre les relacions d'indistinguibilitat. In Proceedings of Primer Congrés Català de Lògica Matemàtica; Universitat Politècnica de Barcelona: Barcelona, Spain, 1982; pp. 51-54.

2. Recasens, J. Indistinguishability Operators: Modelling Fuzzy Equalities and Fuzzy Equivalence Relations. In Studies in Fuzziness and Soft Computing; Kacprzyk, J., Ed.; Springer-Verlag: Berlin/Heidelberg, Germany, 2010; ISBN 978-3-642-16221-3.

3. Klement, E.P.; Mesiar, R.; Pap, E. Triangular norms. In Trends in Logic-Studia Logica Library; Wójcicki, R., Mundici, D., Priest, G., Segerberg, K., Urquhart, A., Wansing, H., Malinowski, J., Eds.; Kluwer Academic Publishers: Dordrecht, The Netherlands, 2000; ISBN 0-7923-6416-3.

4. De Baets, B.; Mesiar, R. Metrics and T-equalities. J. Math. Anal. Appl. 2002, 267, 351-347, doi:10.1006/jmaa.2001.7786.

5. Gottwald, S. On $t$-norms which are related to distances of fuzzy sets. BUSEFAL 1992, 50, 25-30.

6. Hohle, U. Fuzzy equalities and indistinguishability. In Proceedings of the First European Congress on Fuzzy and Intelligent Technologies (EUFIT"93), Eurogress Aachen, Germany, 7-10 September 1993; Zimmermann, H.J. Ed.; ELITE: Aachen, Germany; pp. 358-363.

7. Ovchinnikov, S. Representation of transitive fuzzy relations. In Aspects of Vagueness; Skala, H., Termini, S., Trillas, E., Eds.; Reidel: Dordrecht, The Netherlands, 1984; pp. 105-118.

8. Valverde, L. On the structure of F-indistinguishability operators. Fuzzy Sets Syst. 1988, 17, 313-328, doi:10.1016/0165-0114(85)90096-X.

9. Deza, M.M.; Deza, E. Encyclopedia of Distances, 1st ed.; Springer: Berlin/Heidelberg, Germany, 2009; ISBN 978-3-642-00234-2.

10. Fuster-Parra, P.; Guerrero, J.; Martín, J.; Valero, O. New results on possibilistic cooperative multi-robot system. LNCS 2017, 10451, 21-28, doi:10.1007/978-3-319-66805-5_1.

11. Guerrero, J; Miñana, J.J.; Valero, O.; Oliver, G. Indistinguishability operators applied to task allocation problems in multi-agent systems. Appl. Sci. 2017, 7, 963, doi:10.3390/app7100963. 
12. Gerrero, J.; Miñana, J.J.; Valero, O. A comparative analysis of indistinguishability operators applied to swarm multi-robot task allocation problem. LNCS 2017, 10451,1-9, doi:10.1007/978-3-319-66805-5_3.

13. Gregori, V.; Morillas, S.; Sapena, A. Examples of fuzzy metrics and applications. Fuzzy Sets Syst. 2011, 170, 95-111.

14. Morillas, S.; Gregori, V.; Peris-Fajarnés, G; Latoree, P. A fast impulsive noise color image filter using fuzzy metrics. Real-Time Imaging 2005, 11, 417-428.

15. Morillas, S.; Gregori, V.; Peris-Fajarnés, G. New adaptative vector filter using fuzzy metrics. J. Electron. Imaging 2007, 16, 1-15.

16. Kramosil, I.; Michalek, J. Fuzzy metric and statistical metric spaces. Kybernetika 1975, 11, 336-344.

17. Grabiec, M. Fixed points in fuzzy metric spaces. Fuzzy Sets Syst. 1988, 27, 385-389, doi:10.1016/0165-0114(88)90064-4.

18. George, A.; Veeramani, P. On some results in fuzzy metric spaces. Fuzzy Sets Syst. 1994, 64, 395-399, doi:10.1016/0165-0114(94)90162-7.

19. Gregori, V.; Morillas, S.; Sapena, A. A class of completable fuzzy metric spaces. Fuzzy Sets Syst. 2010, 161, 2193-2205.

20. Chistyakov, V.V. Modular metric spaces, I: Basic concepts. Nonlinar Anal-Theor. 2010, 72, 1-14, doi:10.1016/j.na.2009.04.057.

(C) 2017 by the authors. Licensee MDPI, Basel, Switzerland. This article is an open access article distributed under the terms and conditions of the Creative Commons Attribution (CC BY) license (http:/ / creativecommons.org/licenses/by/4.0/). 